\title{
Levels of molecular angiogenic and antiangiogenic in pregnant women with risk of preeclampsia
}

\author{
Diah Wulandari' ${ }^{1 *}$, Mohamad Sulchan ${ }^{2}$, Syarief Thaufik Hidayat ${ }^{3}$
}

\author{
${ }^{1}$ Department of Midwifery, Universitas Gadjah Mada, Yogyakarta, Indonesia \\ ${ }^{2}$ Departement of Nutrition Science, Faculty of Medicine, Universitas Diponegoro, Semarang, Indonesia \\ ${ }^{3}$ Departement of Obstetrics and Gynecology, Faculty of Medicine, Universitas Diponegoro, Semarang, Indonesia
}

Received: 02 August 2019

Revised: 03 September 2019

Accepted: 11 September 2019

\section{*Correspondence:}

Dr. Diah Wulandari,

E-mail: diah_wulandari@ugm.ac.id

Copyright: () the author(s), publisher and licensee Medip Academy. This is an open-access article distributed under the terms of the Creative Commons Attribution Non-Commercial License, which permits unrestricted non-commercial use, distribution, and reproduction in any medium, provided the original work is properly cited.

\begin{abstract}
Background: Angiogenic and antiangiogenic imbalances play a major role in the pathogenesis of preeclampsia. Increased production of sFlt-1 by the placenta causes free circulating PIGF and VEGF concentrations to lower because it is bound by sFlt-1. Measuring levels of angiogenic and antiangiogenic proteins as biomarkers indicates placental dysfunction and distinguishes preeclampsia from other disorders. This study aims to analyze the levels of angiogenic and antiangiogenic molecules in pregnant women at risk for preeclampsia.

Methods: The study with a cross-sectional design was carried out in 11-15 weeks gestational age whom had a risk of preeclampsia with 30 samples in primary health care starting April-August 2018. Blood serum was measured by molecular levels of VEGF, PlGF, sFlt-1, and sFlt-1/PIGF ratio using the ELISA method. Data analysis used Pearson product moment test.

Results: The mean of VEGF levels are $15.5 \pm 21.6$, PlGF $89.7 \pm 55.5$, sFlt-1 $11519.4 \pm 5126.0$ and the ratio sFlt-1/PlGF 166.7 \pm 102.1 . Correlation value of risk factors for preeclampsia with molecular levels of VEGF $r=-0.05 ; p=0.76$, PlGF $r=-0.21 ; p=0.26$, sFlt $r=0.01 ; p=0.99$ and ratio sFlt-1/PIGF $r=0.10 ; p=0.58$.

Conclusions: The higher the total score of preeclampsia risk factor, the lower the molecular level of VEGF and PIGF is. Moreover, the higher the total score of preeclampsia risk factor, the higher the molecular level sFlt-1 and the sFlt1/PIGF ratio is. There are no significant correlation between total score of preeclampsia risk factor and levels of molecule VEGF, PlGF, sFlt-1 and sFlt-1/PlGF ratio.
\end{abstract}

Keywords: Preeclampsia, PlGF, Risk factors, sFlt-1, VEGF

\section{INTRODUCTION}

Preeclampsia is a specific condition in pregnancy which is characterized by the presence of placental dysfunction and maternal response due to systemic inflammation along with endothelial activation and coagulation. ${ }^{1}$ The reported incidence of preeclampsia in 2013 in Indonesia was $128,273 /$ year or estimated around $5.3 \%$. Maternal mortality caused by preeclampsia was reported to be
29,000 and as many as $25.8 \%$ of the Indonesian population aged more than 18 years old were suffered from hypertension, whereas $6-10 \%$ of them were happened during pregnancy. ${ }^{2}$

Preeclampsia is indeed called as a "disease of theories," because the pathogenesis of preeclampsia has not been well understood. Some of these theories are placental ischemia, oxidative stress and free radicals, and the 
theory of VLDL, maladaptive immune, and imbalance of angiogenic and antiangiogenic factors. ${ }^{3-5}$ Those the theory of angiogenic and antiangiogenic factors play major role in the pathogenesis of preeclampsia.,

The clinical signs of preeclampsia appear after the $20^{\text {th }}$ weeks of pregnancy, however the ischemia and pathogenic processes has begun in the first trimester of the previous few weeks. ${ }^{4}$ Should preeclampsia not well diagnosed or treated, it can cause multiorgan failure, coagulation, eclampsia and even maternal and fetal death. ${ }^{8}$ The National Institute for Health and Care Excellence (NICE) recommended that the high risk of preeclampsia is most effectively identified in the $11-13^{\text {th }}$ weeks of pregnancy in order to make early primary prevention and intervention management. ${ }^{1,9}$

Risk factors for preeclampsia were assessed through anamnesis as such: age $>40$ years, nullipara, multiparous with a history of previous preeclampsia, multiparas with pregnancies by new partners, multiparas who had a previous pregnancy 10 years or more, history of preeclampsia in mothers or sisters, multiple pregnancies, diabetes mellitus, chronic hypertension, kidney disease, pregnancy with insemination of sperm, oocyte or embryo donors, obesity before pregnancy, infection during pregnancy, autoimmune diseases such as systemic lupus erythematosis (SLE) and antiphospholipid syndrome (APS). ${ }^{1,2}$ Risk factors for pregnant women above can develop into preeclampsia in advanced pregnancy. ${ }^{10}$

Some risk factors condition for preeclampsia can cause placental hypoxia. Furthermore this matter triggers the releasing of some markers of placental hypoxia. In the form of increased secretion of sFlt-1 and sFlt-1 serum enter the maternal systemic circulation. Lack of placental perfusion is the main cause of injury to ischemic perfusion in the placenta therefore that all of these processes cause symptoms of preeclampsia. ${ }^{9}$ Increased production of sFlt-1 by placenta preeclampsia causes circulating of free PIGF and VEGF concentrations become low, because it is bound by sFlt-1. ${ }^{10}$ Decreasing levels of VEGF and PIGF can cause neovascularization during placentation. Following the result in failure to form normal uteroplacental circulation. As known, the failure indicates infarction of the placenta, arteriosclerosis, superficial cytotrophoblast invasion, and inadequate remodeling of the uterine spiral arteries. ${ }^{11}$

This study aims to analyze the levels of angiogenic and antiangiogenic molecules in pregnant women at risk for preeclampsia and correlation of total score of preeclampsia risk factor with VEGF, PlGF, sFlt-1 and sFlt-1/PlGF ratios.

\section{METHODS}

This study used a cross sectional design, involving subjects who were 11-15 weeks pregnancy whom had a risk of preeclampsia with 30 samples. Sample size was calculated by obtaining the prevalence of pregnant women whom had a risk of preeclampsia in primary health care. This research was conducted in 10 primary health care facilities, Sleman Yogyakarta, Indonesia starting April-August 2018. The inclusion criteria are first and second trimester pregnant women (gestational age 11-15 weeks), having one of the several high risk of preeclampsia including: multiparas with a history of previous preeclampsia, multiple pregnancy, diabetes melitus (DM), chronic hypertension, kidney disease, antiphospholipid syndrome (APS), and Systemic Lupus Erythematous (SLE) autoimmune disease) or have more than 2 risks of preeclampsia: nullipara, obesity before pregnancy $\left(\mathrm{BMI}>25 \mathrm{~kg} / \mathrm{m}^{2}\right)$, age $>40$ years, multiparas who have a previous pregnancy 10 years or more, history of preeclampsia in mothers or sisters, multiparous with pregnancy by a new partner, diastolic blood pressure> $80 \mathrm{mmHg}$ (MAP> 100. Exclusion criteria are: suffering from infectious diseases, anemia (Hb levels <11gm\%), and mothers were on insulin therapy.

Primary data collection begun with anamnesis, physical examination and laboratory examination (urine protein and haemoglobin levels) as a risk screening for preeclampsia.

Each participant measured the total score of preeclampsia risk factor and the levels of VEGF, PlGF, sFlt-1, and sFlt-1/ PIGF ratio. The total score was obtained from the number of risk factor characteristics possessed by each participant multiplied by the weight score of each of the risk factor (Table 1). Participants were measured for blood serum to determine levels of VEGF, PlGF, sFlt-1, and sFlt-1/ PlGF ratio.

Confounding variable includes the immune system, hypoxia and genetics and has been controlled by restriction through the selection of inclusion and exclusion criteria.

Data analysis using Pearson product moment to find out the relationship between the two variables.

VEGF, PlGF and sFlt-1 measurement were done by ELISA and using reagents Quantikine Human VEGF Immunoassay reagents, $C$ at ( $\mathrm{R}$ and $\mathrm{D}$ systems). No DVE00), Quantikine Human PlGF Immunoassay reagents, ( $\mathrm{R}$ and $\mathrm{D}$ systems) Cat. No DPG00), and Quantikine Human VEGF R1 / Flt-1 Immunoassay, (R and D systems) Cat reagents. DVR No. 100B), respectively. Informed consent was obtained from all study participants.

\section{RESULTS}

Most of risk factors for preeclampsia in pregnant women are BMI $\geq 25 \mathrm{~kg} / \mathrm{m}^{2}$, which is $86.7 \%$. The highest score weighting risk factor for preeclampsia is 4 in pregnant women who have hypertension (Table 1). 
Some participants have more than 1 risk factor for preeclampsia. The total score was obtained from the number of risk factor characteristics possessed by each participant multiplied by the weight score of each of the risk factor). Total preeclampsia risk factor scores $2,3,4,5$, and 6 were 8 (26.7\%), $9(30 \%), 8(26.7 \%), 3(10 \%)$ and $2(6.7 \%)$.

Table 1 Overview of characteristics of pregnant women and preeclampsia risk factor scores.

\begin{tabular}{|lll|}
\hline Risk factor of preeclampsia & (Weighted score of risk factor) & n $(\%)(\mathbf{n = 3 0})$ \\
\hline Age $>40$ years old & 1 & $3(10 \%)$ \\
\hline Primigravida & 2 & $8(16.7 \%)$ \\
\hline BMI $\geq 25 \mathrm{~kg} / \mathrm{m}^{2}$ & 2 & $26(86.7 \%)$ \\
\hline History of preeclampsia: & 3 & $4(13.3 \%)$ \\
\hline Pregnancy intervals $>10$ years & 1 & $2(6.7 \%)$ \\
\hline Family history & 1 & $7(23.3 \%)$ \\
\hline Hypertension & 4 & $2(6.7 \%)$ \\
\hline Diabetes mellitus & 2 & $1(3.3 \%)$ \\
\hline
\end{tabular}

Table 2: Correlation of total score of preeclampsia risk factor with VEGF, PIGF, sFlt-1 and sFlt-1/ PIGF ratios.

\begin{tabular}{|llll|}
\hline Levels of molecular & Mean \pm SD & $r$ & p-value \\
\hline VEGF & $15.5 \pm 21.6$ & -0.05 & $0.76^{\mathrm{a}}$ \\
\hline PlGF & $89.7 \pm 55.5$ & -0.21 & $0.26^{\mathrm{a}}$ \\
\hline sFlt-1 & $11519.4 \pm 5126.0$ & 0.01 & $0.99^{\mathrm{a}}$ \\
\hline Rasio sFlt-1/ PlGF & $166.7 \pm 102.1$ & 0.10 & $0.58^{\mathrm{a}}$ \\
\hline
\end{tabular}

${ }^{a}$ Pearson product moment.

Then a correlation analysis was performed between the total score of preeclampsia risk factor with the levels of molecular VEGF, PlGF, sFlt-1 and sFlt-1/ PlGF ratio, results in Table 2.

The mean VEGF levels are 15.5 \pm 21.6 , PlGF 89.7 \pm 55.5 , sFlt-1 $11519.4 \pm 5126.0$ and the ratio sFlt-1/ PlGF $166.7 \pm 102.1$ (Tabel 2).

Based on the weight of risk factors and the calculation of the total score of preeclampsia risk factors, the results showed that there are no significant correlation between total score of preeclampsia risk factor and levels of molecular VEGF, PIGF, sFlt-1 and sFlt-1/ PlGF ratio. However, there is a negative correlation on the level of VEGF and PlGF molecules and a positive correlation on the level of the sFlt-1 molecule and the sFlt-1/ PlGF ratio. The higher the total score of preeclampsia risk factor the lower the levels of VEGF and PIGF molecules, the higher the total score of preeclampsia risk factor the higher the level of sFlt-1 molecule and sFlt-1/ PIGF ratio.

\section{DISCUSSION}

This study shows that the characteristics of the research subjects are risk factors for preeclampsia. Most of risk factors for preeclampsia in pregnant women are BMI $\geq$ $25 \mathrm{~kg} / \mathrm{m}^{2}$, which is $86.7 \%$ (Table 1). Risk factors for pregnant women above are at risk of developing preeclampsia in advanced pregnancy. These risk factors, which have been identified, can help in assessing the risk of pregnancy at the initial antenatal. ${ }^{10}$

\section{Risk factor of preeclampsia}

Primiparity is significantly associated with the incidence of preeclampsia with a risk of increasing 2x (OR2.39; 1.23-4.65) compared to multiparity. ${ }^{11}$ In primiparity there is a reaction of maternal adaptation to trophoblast invasion in early pregnancy. Normal invasion failure of trophoblast cells causes maladaptation of the spriral arteries, which is associated with the causes of preeclampsia. ${ }^{12}$ Based on Dekker's research in Esplin et al, it was stated that first or multiparous pregnancies with new partners showed that there were interactions between maternal antibodies and fetal antigens derived from paternal genes, where father's genetic contribution to fetal genotypes could contribute to the development of preeclampsia. ${ }^{13}$

Based on the Duckitt report, 2005, preeclampsia occurred doubled at the age of mothers over 40 years. The risk of preeclampsia in the second pregnancy increases with the age of the mother (1.3 times every 5 years of age $).{ }^{14}$ The more mature a woman in age, the higher the risk factors will be, which may be caused by an increase in the reaction of the villi which leads to the occurrence of preeclampsia. ${ }^{11}$ 
Obesity increased the risk of preeclampsia by 2.47 times (95\% CI, 1.66-3.67), whereas women with BMI before pregnancy $>35$ compared with BMI 19-27 had a fourfold risk of preeclampsia $(95 \% \mathrm{CI}, 3.52-5.49) .{ }^{14}$ Obesity leads to lipotoxic conditions characterized by decreased angiogenic regulation and increased pro-inflammatory cytokines. Proinflammatory cytokines, especially TNF- $\alpha$ through the process of apoptosis can limit the invasion of cytotrophoblast cells into the spiral arteries. ${ }^{15}$ Increased triglyceride levels in obesity due to the production of VLDL in the liver because of the increase in free fatty acids entering the liver. This causes oxidative cell regeneration resulting in endothelial cell dysfunction and causing preeclampsia. Obesity causes changes in the body's metabolism in the form of increased inflammation of the cardiovascular system, oxidative stress and decreased PIGF. The high level of fat reserves increases leptin levels and decreases in adiponectin which causes insulin resistance, endothelial dysfunction and impaired vasodilation in order that increased blood pressure vasoconstriction interferes with the distribution of nutrients to the fetus. ${ }^{16,17}$

The history of preeclampsia is a major risk factor or high risk. Based on the research of Cincotta RB and Arngrimsson $\mathrm{R}$ in Esplin et al, showed that in primigravidas with a family history of preeclampsia, the incidence of preeclampsia increased threefold compared to primigravida without a family history. Other studies also mention the risk of increasing the prevalence of preeclampsia and eclampsia significantly higher in children of women with families with a history of preeclampsia $(23 \%)$ than in daughter-in-law $(10 \%){ }^{13}$ According to Duckit and Hanington, 2005 the risk increased by 7-fold (RR 7.19 95\% CI 5.85-8.83). Pregnancy in women with a history of preeclampsia is associated with a high incidence of severe preeclampsia, early onset preeclampsia, and poor perinatal effects. ${ }^{14}$

A study involving 760,901 women in Norway, showed that multiparous women with a previous pregnancy distance of 10 years or more had a risk of preeclampsia about the same as nullipara. ${ }^{14}$ Robillard et al reported that the risk of preeclampsia increased according to the length of the interval with the first pregnancy ( 1.5 times every 5 years the distance of the first and second pregnancies). NICE reports that the $<10$ year pregnancy interval does not increase the risk of recurrent preeclampsia, but some reports suggest an increased risk of recurrent preeclampsia at $<2$ years or $>10$ years of pregnancy. ${ }^{18}$

A history of family preeclampsia also increases the risk almost 3-fold (RR 2.90 95\% CI 1.70-4.93). The history of maternal preeclampsia increases risk by 3.6 times (RR $3.695 \%$ CI 1.49-8.67). ${ }^{14}$ In the previous study it was found $26 \%$ of the incidence of preeclampsia in girls and women with preeclampsia but only $8 \%$ of incidents in daughter-in-law. The increasing prevalence of preeclampsia in girls born to preeclamptic mothers, associated with non-preeclamptic pregnancies from the same mother, may indicate a fetal genotypic influence on the reliability of preeclampsia. ${ }^{19}$

Shibuya et al, conducted a study on a population of 56,968 , indicating that the risk of preeclampsia increased almost four-fold if diabetes occurred before pregnancy (RR 3.56; 95\% CI 2.54 - 4.99). ${ }^{14}$ Chappell et al, study of 861 women with chronic hypertension, found an incidence of superimposed preeclampsia of $22 \%$ and almost half of those with early onset preeclampsia $(<34$ weeks) with worse maternal and perinatal output. ${ }^{10}$ Karumanchi et al, said that women with DM and hypertension increased the incidence of preeclampsia and also showed the presence of several factors in the maternal risk of preeclampsia. In addition to increased vascular reactivity, vasoconstriction is mediated in part by changes in local concentrations of several vasoactive molecules, including vasoconstrictor norepinephrine, endothelin, and thromboxane, and vasodilator prostacyclin and nitric oxide. ${ }^{18}$

\section{Angiogenic and antiangiogenic in pregnant women with risk of preeclampsia}

Pregnant women at risk of preeclampsia cause changes in levels of VEGF, PIGF, sFlt-1 and the ratio of sFlt-1/ PIGF levels, have low levels of angiogenic molecules, and high antiangiogenic levels (Table 2). This study showed that the VEGF and PIGF correlation values showed a negative correlation, namely the higher the total score of preeclampsia risk factor the lower the levels of VEGF and PIGF molecules. While the sFlt-1 correlation value and sFlt-1 / PlGF ratio showed a positive correlation, namely the higher the total score of preeclampsia risk factor the higher the level of sFlt-1 molecule and sFlt-1/ PlGF ratio. There are no significant correlation between total score of preeclampsia risk factor and levels of molecul VEGF, PIGF, sFlt-1 and sFlt-1/ PlGF ratio (Table 2).

The pathogenic process of preeclampsia begins during the first trimester, long before clinical signs appear clearly. ${ }^{4}$ In the early stages of the pathogenesis of preeclampsia there is a decrease in placental perfusion, asymptomatic (placental). This decrease is characterized by abnormal placentation, followed by elaboration of certain soluble factors that enter the maternal circulation and cause widespread development of endothelial dysfunction. ${ }^{6}$ At this stage, there is a decrease in VEGF which will lead to disruption of endothelization and invasion of the spiral arteries to the myometrium, leading to increased vascular resistance, this will be followed in the second stage, where ischemia occurs in the placenta, causing oxidative stress on the placenta and releasing proteins such as sFlt- 1, prostaglandin, and cytokines. ${ }^{20}$

Measuring the levels of angiogenic and antiangiogenic proteins in the bloodstream as biomarkers indicates placental dysfunction and differentiates preeclampsia from other disorders, such as gestational hypertension 
and chronic glomerulonephritis. ${ }^{4}$ Angiogenic factors (VEGF, PIGF) correlate with disease severity, can be detected several weeks before clinical presentation of the disease and have a predictive value for the diagnosis of early onset preeclampsia. ${ }^{21}$

\section{Vascular endothel growth factor}

VEGF is bound by sFlt-1 in preeclampsia, resulting in low levels of circulating free VEGF. ${ }^{22}$ VEGF induces vasculogenesis and angiogenesis and plays an important role in endothelial cell proliferation. VEGF inactivation can cause lethal effects on the embryon and vascular defects in the placenta. Decreased VEGF levels due to binding with receptors in the circulating increase indicate angiogenic and antiangiogenic imbalances which subsequently trigger endothelial dysfunction. ${ }^{23}$ This results in failure to form normal uteroplacental circulation. The failure indicates infarction of the placenta, arteriosclerosis, superficial cytotrophoblast invasion, and inadequate remodeling of the uterine spiral arteries. $^{20}$

\section{Placental growth factor}

Several studies have shown the important role of PIGF in regulating angiogenesis under pathological. ${ }^{24}$ In women who subsequently experience preeclampsia, serum PIGF concentrations do not increase when compared to the age of 10-13 weeks of pregnancy and experience a significant decrease 5 weeks before the appearance of clinical manifestations. ${ }^{25}$ Serum PlGF examination at 11-13 weeks pregnancy can identify a high proportion of highrisk pregnancies for early onset PE26. PIGF concentrations begin to decrease 9-11 weeks before hypertension and urine protein are seen, with an increase of 5 weeks before the onset. ${ }^{25}$

\section{Soluble fms like tirosine kinase-1}

Increased expression of sFlt-1 can interfere with trophoblast function and endothelial cells in preeclampsia. ${ }^{27}$ An increase in sFlt-1 in the serum of pregnant women is associated with endothelial dysfunction that occurs in preeclampsia, which is characterized by the emergence of clinical manifestations. ${ }^{28}$ Levels of sFlt-1 in the serum of pregnant women with preeclampsia are high at 20 weeks' gestation and increase significantly within 5 weeks before hypertension and preeclampsia arise. ${ }^{29}$

The sFlt-1 / PlGF $\leq 38$ ratio can be used to predict the absence of short-term preeclampsia in women with suspected clinical syndromes. Preeclampsia often occurs in patients with a ratio of sFlt- $1 /$ PlGF ratio $\geq 85(40.5 \%)$ compared to 33 to $85(28.1 \%)$ and $<33(9.8 \%)$. The Zeisler et al, study found that the sFlt-1 / PlGF ratio with a cutoff of 38 had an important predictive value. ${ }^{30}$
The strengths of this study is to analyze the angiogenic levels of VEGF and PlGF, antiangiogenic sFlt-1 and sFlt$1 / \mathrm{PlGF}$ ratios in pregnant women with the risk of preeclampsia measured in one study of the four measured markers which describe changes that can occur in spiral arteries (imbalance theory pathway angiogenic and anti angiogenic factors).

\section{CONCLUSION}

There are no significant correlation between total score of preeclampsia risk factor and levels of molecul VEGF, PlGF, sFlt-1 and sFlt-1/ PlGF ratio.

The higher the risk factor score, the lower the molecular level of VEGF and PIGF will be. Furthermore, the higher the total score of preeclampsia risk factor, the higher the molecular level sFlt-1 and the sFlt-1 / PlGF ratio is.

\section{Recommendations}

Based on the results of the study, recommendations that can be suggested are: Early risk factors detection and screening for preeclampsia in $<16$ weeks of gestation is very necessary, the need of regular monitoring of weight gain during pregnancy based on pregnancy BMI, especially in pregnant women with BMI $>25 \mathrm{~kg} / \mathrm{m}^{2}$ and advanced researchers to be able to prove the circulation of antiangiogenic and angiogenic levels in each trimester in pregnant women with risk factors for preeclampsia pregnancy and in normal pregnancy.

\section{ACKNOWLEDGMENTS}

Authors would like to thank all the investigators and all the study participants.

Funding: No funding sources

Conflict of interest: None declared

Ethical approval: The study was approved by the Institutional Ethics Committee

\section{REFERENCES}

1. Magee L, Pels A, Helewa M, Rey E, von Dadelszen P. Diagnosis, evaluation, and management of the hypertensive disorders of pregnancy: executive summary. J Obs Gynaecol Can. 2014;36(5):416-38.

2. Wibowo N, Irwinda R, Frisdiantiny E, Kartaka M, Mose JC, Chalid MT, et al. Pedoman nasional pelayanan kedokteran diagnosis dan tata laksana preeklampsia. Jakarta: Perkumpulan Obstetri dan Ginekologi Indonesia Himpunan Kedokteran Feto Maternal. 2016:11-20.

3. Uzan J, Carbonnel M, Piconne O, Asmar R, Ayoubi JM. Pre-eclampsia: Pathophysiology, diagnosis, and management. Vasc Health Risk Manag. 2011;7(1):467-74.

4. Gathiram P, Moodley J. Pre-eclampsia: its pathogenesis and pathophysiolgy. Cardiovasc J Afr. 2016;27(2):71-8. 
5. Esta M, Afdal P, Anggraini NW. Malondialdehyde levels are higher and glutathione levels are lower in preeclampsia than in normal pregnancies. Univ Med. 2017;36(3):179-86.

6. Lam C, Lim KH, Karumanchi SA. Circulating angiogenic factors in the pathogenesis and prediction of preeclampsia. Hypert. 2005;46(5):1077-85.

7. Levine RJ, Lam C, Qian C, Yu KF, Maynard SE, Sachs $\mathrm{BP}$, et al. Soluble endoglin and other circulating antiangiogenic factors in preeclampsia, 2006. Available at:

http://www.nejm.org/doi/pdf/10.1056/NEJMoa055352. Accessed on $2^{\text {nd }}$ April 2019.

8. Thadhani RI, Johnson RJ, Karumanchi SA. Hypertension during pregnancy: a disorder begging for pathophysiological support. Hypert. 2005;46(6):1250-1.

9. National Institute for Health and Care Excellence. Preterm labour and birth, 2015. Available at: http://nice.org.uk/guidance/ng25. Accessed on $2^{\text {nd }}$ April 2019.

10. Chappell LC, Enye S, Seed P, Briley AL, Poston L, Shennan AH. Adverse perinatal outcomes and risk factors for preeclampsia in women with chronic hypertension: A prospective study. Hypert. 2008;54:1002-9.

11. Verma MK, Kapoor P, Yadav R, Manohar RK. Risk factor assessment for preeclampsia: a case control study. Int J Med Public Heal. 2017;7(3):172-7.

12. Agrawal S, Millett C, Casas JP, Walia GK, StainesUrias E. Prevalence of and risk factors for eclampsia in pregnant women in India. Fam Med Community Heal. 2017;5(4):225-44.

13. Esplin S, Ausett M, Fraser A, Kerber R, Mineau G, Carillo J, et al. Paternal and maternal components of the predisposition to preeclampsia. $\mathrm{N}$ Engl $\mathrm{J}$ Med. 2002;346(15):1105-12.

14. Duckitt K. Risk factors for pre-eclampsia at antenatal booking: systematic review of controlled studies. BMJ. 2005;330(7491):565.

15. Dekker G, Robillard PY. The birth interval hypothesis Does it really indicate the end of the primipaternity hypothesis. J Reprod Immunol. 2003;59(2):245-51.

16. Cunningham FG. Williams obstetrics. $24^{\text {th }}$ edition. F. Gary Cunningham, Kenneth J. Leveno, Steven L. Bloom, Catherine Y. Spong, Jodi S. Dashe, Barbara L. Hoffman, Brian M. Casey JSS, editor. United States Publisher: New York: M: New York: McGraw-Hill Education/Medical; 2014.

17. Weiss JL, Malone FD, Emig D, Ball RH, Nyberg DA, Comstock $\mathrm{CH}$, et al. Obesity, obstetric complications and cesarean delivery rate - A population-based screening study. Am J Obstet Gynecol. 2004;190(4):1091-7.

18. Sukhatme VP, Stillman IE, Epstein FH, Karumanchi SA, Maynard SE. Preeclampsia: a renal perspective. Kidney Int. 2005;67(6):2101-13.
19. Sibai BM. Diagnosis and management of gestational hypertension and preeclampsia. Obstet Gynecol. 2003;102(1):181-92.

20. Chen Y. Novel angiogenic factors for predicting preeclampsia: sFlt-1, PlGF, and soluble Endoglin. Open Clin Chem J. 2009;2:1-6.

21. Nikuei P, Malekzadeh K, Rajaei M, Nejatizadeh A, Ghasemi N. The imbalance in expression of angiogenic and anti-angiogenic factors as candidate predictive biomarker in preeclampsia. Iran $\mathrm{J}$ Reprod Med. 2015;13(5):251-62.

22. Agarwal R, Bills JE, Hecht TJW, Light RP. Role of home blood pressure monitoring in overcoming therapeutic inertia and improving hypertension control: A systematic review and meta-analysis. Hypert. 2011;57(1):29-38.

23. Powe CE, Levine RJ, Karumanchi SA. Preeclampsia, a disease of the maternal endothelium: The role of antiangiogenic factors and implications for later cardiovascular disease. Circulation. 2011;123(24):2856-69.

24. Sibiude J, Guibourdenche J, Dionne MD, Le Ray C, Anselem O, Serreau R, et al. Placental Growth Factor for the prediction of adverse outcomes in patients with suspected preeclampsia or intrauterine growth restriction. PLoS One. 2012;7(11):e50208.

25. Levine RJ, Maynard SE, Qian C, Lim KH, England LJ, $\mathrm{Yu} \mathrm{KF}$, et al. Circulating angiogenic factors and the risk of preeclampsia. N Engl J Med. 2004;350(7):672-83.

26. Poon LC, Nicolaides KH. Early Prediction of Preeclampsia. Obstet Gynecol Int. 2014;2014(2):1-11.

27. Shibuya M. Involvement of Flt-1 (VEGF receptor-1) in cancer and preeclampsia. Proc Japan Acad Ser B. 2011;87(4):167-78.

28. Maynard S, Epstein FH, Karumanchi SA. Preeclampsia and angiogenic imbalance. Annu Rev Med. 2008;59(1):61-78.

29. Yang Gu, Lewis YW. Placental productions and expressions of soluble Endoglin, soluble fms-Like tyrosine kinase receptor-1, and placental growth factor in normal and preeclamptic pregnancies. J Clin Endocrinol Metab. 2008;93(1):260-6.

30. Zeisler H, Llurba E, Chantraine F, Vatish M, Staff AC, Sennström M, et al. Predictive value of the sFlt-1:PlGF ratio in women with suspected preeclampsia. Obstet Gynecol Surv. 2016;71(5):273-4.

Cite this article as: Wulandari $\mathrm{D}$, Sulchan $\mathrm{M}$, Hidayat ST. Levels of molecular angiogenic and antiangiogenic in pregnant women with risk of preeclampsia. Int J Reprod Contracept Obstet Gynecol 2019;8:4034-9. 